\title{
Modeling Enclosures for Large-Scale Superconducting Quantum Circuits
}

\author{
P.A. Spring $\odot,{ }^{*}$ T. Tsunoda, B. Vlastakis, and P.J. Leek $\odot$ \\ Clarendon Laboratory, Department of Physics, University of Oxford, Oxford OX1 3PU, United Kingdom
}

(Received 30 October 2019; revised 22 May 2020; accepted 16 July 2020; published 21 August 2020)

\begin{abstract}
Superconducting quantum circuits are typically housed in conducting enclosures in order to control their electromagnetic environment. As devices grow in physical size, the electromagnetic modes of the enclosure come down in frequency and can introduce unwanted long-range cross-talk between distant elements of the enclosed circuit. Incorporating arrays of inductive shunts such as through-substrate vias or machined pillars can suppress these effects by raising these mode frequencies. Here, we derive simple, accurate models for the modes of enclosures that incorporate such inductive-shunt arrays. We use these models to predict that cavity-mediated interqubit couplings and drive-line cross-talk are exponentially suppressed with distance for arbitrarily large quantum circuits housed in such enclosures, indicating the promise of this approach for quantum computing. We find good agreement with a finite-element simulation of an example device containing more than 400 qubits.
\end{abstract}

DOI: 10.1103/PhysRevApplied.14.024061

\section{INTRODUCTION}

Superconducting circuits are a promising platform for quantum computing. Their success originates partly from the intrinsically strong coupling of superconducting qubits to electromagnetic fields, which itself derives from their macroscopic size. This strong coupling facilitates fast quantum logic gates [1,2], readout [3], and reset of qubits [4]. However, it also makes superconducting qubits prone to couple to spurious electromagnetic (EM) modes in their environment. This can cause deleterious effects such as radiative energy relaxation [5], coherent leakage of the qubit state [6], and mediation of undesired interqubit couplings [7]. It is therefore important to engineer their environment such that couplings to spurious EM modes are suppressed. A powerful solution is to house quantum circuits in a cavity that has a fundamental (lowest) mode frequency well above qubit frequencies [8].

As superconducting circuits grow in size, the fundamental frequency of a simple contiguous cavity enclosure must come down. This can be mitigated to some degree by designing vacuum regions into the cavity that reduce the field energy stored in the high dielectric substrate on which the circuit is fabricated $[9,10]$, but this approach is not scalable. An approach that is known to be scalable is to inductively shunt the cavity with an array of throughsubstrate vias (TSVs) [11-13], which must be bonded in some way to both sides of the cavity. The scalability of this approach is related to the physics of metallic photonic crystals $[14,15]$, which predicts a scale-independent cutoff

\footnotetext{
*peter.spring@physics.ox.ac.uk
}

frequency for this type of periodic metal structure, below which it cannot sustain modes [14].

This scale-independent cutoff frequency means inductively shunted cavities can provide a clean EM environment to superconducting circuits at arbitrary size. When engineering the layout of the shunt array, it is then relevant to ask how the cavity-mode frequencies depend on the shunt spacing and size, and how cavity-mediated interqubit couplings and drive-line cross-talk is affected.

The purpose of this work is to address these questions, by constructing accurate, closed-form, physically intuitive models for the modes of a cavity that is inductively shunted by a square array of cylinders; and to then use these results to predict cavity-mediated interqubit couplings and driveline cross-talk inside such a cavity.

The paper is arranged as follows: in Sec. II we develop a plasma model and a circuit model for cavities with periodic inductive shunt arrays. In Sec. III we use these models to predict interqubit coupling and drive-line cross-talk for superconducting qubits inside such cavities. In Sec. IV we test the predictions against a finite-element (FE) simulation of a simplified device containing a $21 \times 21$ grid of qubits, representing a device in the noisy intermediate scale quantum regime [16].

\section{MODELS FOR THE PERIODICALLY INDUCTIVELY SHUNTED CAVITY}

In Fig. 1, we illustrate how the fundamental frequency of a simple rectangular cavity [Fig. 1(a)] is altered by the presence of a protruding pillar. An unshorted pillar behaves as a capacitive shunt [Fig. 1(b)], decreasing the cavitymode frequency, whereas a shorted pillar behaves as an 


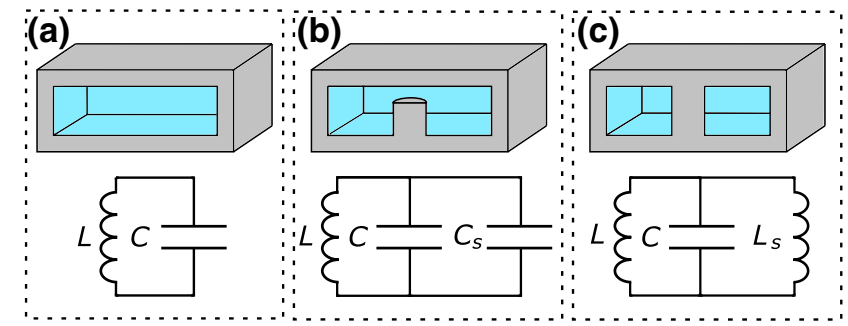

(d)

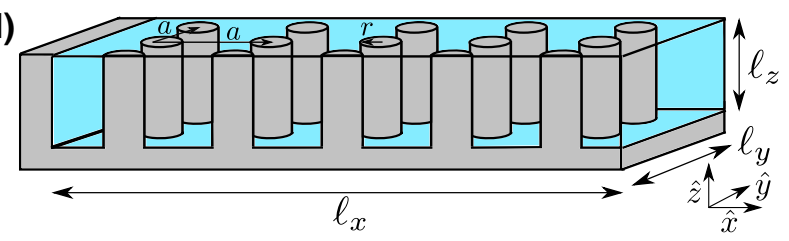

FIG. 1. (a)-(c) Cross sections of rectangular cavities along with circuit representations of their fundamental mode. (d) A cavity filled with a dielectric and inductively shunted by a square array of conducting cylinders, spacing $a$, and radius $r$.

inductive shunt [Fig. 1(c)], increasing the cavity-mode frequency.

We consider the extension of the single inductive shunt to an inductive shunt array, as shown in Fig. 1(d). The array is formed of cylinders radius $r$ and equal spacing $a$ in $\hat{\mathbf{x}}$ and $\hat{\mathbf{y}}$. The cavity is perfectly conducting, has dimensions $\ell_{x}, \ell_{y}, \ell_{z}$, and is filled with a uniform material with dielectric permittivity $\epsilon_{r}$. Multiple dielectric layers stacked along the $\hat{\mathbf{z}}$ axis can be modeled simply by an adjustment to $\epsilon_{r}$ (see Appendix A).

In the absence of inductive shunts, the mode frequencies of this cavity are those of a rectangular cavity, given by

$$
f_{n m l}=\frac{1}{2 \sqrt{\epsilon \mu}} \sqrt{\frac{n^{2}}{\ell_{x}^{2}}+\frac{m^{2}}{\ell_{y}^{2}}+\frac{l^{2}}{\ell_{z}^{2}}},
$$

where $n, m, l$ take integer values, and physical solutions permit only one of $n, m, l$ to be zero. In superconducting quantum devices, the circuit substrate thickness does not scale with the circuit size and so we consider the case $\ell_{z} \ll$ $\ell_{x}, \ell_{y}$. The low-frequency spectrum then consists only of $l=0$ modes, which we focus on for the remainder of this paper.

\section{A. Boundary model}

As an illustrative model, consider replacing the array of inductive shunts with a grid of thin conducting walls, with spacing $a$. This results in a fundamental frequency

$$
f_{a}=1 /\left(a \sqrt{2 \epsilon_{0} \epsilon_{r} \mu_{0}}\right) .
$$

This primitive model predicts the existence of a cutoff frequency independent of the total enclosure size, but fails to take into account the shunt radius $r$, which is clearly an oversimplification. In the limit $r \rightarrow 0$ where the shunts disappear, we should instead recover Eq. (1).

\section{B. Plasma model $(r / a<0.1)$}

The behavior of an array of thin, infinitely long conducting cylinders (oriented along $\hat{\mathbf{z}}$ ) has been studied as a metamaterial, and has been shown to behave like an anisotropic plasma in the limit $r \ll a \ll \lambda$, with the associated frequency-dependent permittivity [17]

$$
\epsilon_{\mathbf{p}}(f)=\left[1-\left(\frac{f_{p}}{f}\right)^{2}\right] \hat{\mathbf{z}},
$$

valid for EM waves propagating in the $x-y$ plane [18]. The plasma frequency $f_{p}$ is accurately predicted by a simple function of the cylinder radius and spacing $[19,20]$

$$
\begin{aligned}
f_{p} & =\frac{f_{a}}{\sqrt{\pi}\left[\ln \left(\frac{a}{r}\right)-\Pi\right]^{0.5}} \\
\Pi & =\ln (2 \pi)-\pi / 6-\Sigma_{n=1}^{\infty}[\operatorname{coth}(n \pi)-1] / n \approx 1.31 .
\end{aligned}
$$

We wish to apply these equations to the $l=0$ modes of our inductively shunted cavity. These modes are formed by EM waves propagating in the $x-y$ plane, and have their electric field oriented along $\hat{\mathbf{z}}$, for which Eq. (3) applies. Additionally, these modes have EM fields, which are independent of $\ell_{z}$, and so we do not require $\ell_{z} \rightarrow \infty$. Thus we can apply Eqs. (3) and (4) to the $l=0$ modes of our inductively shunted cavity.

If we define $f_{n m}$ as the $l=0$ mode frequencies of the cavity without the shunt array, and $f_{n m}^{\prime}$ as the frequencies with the array, then we expect

$$
f_{n m}^{\prime}=\frac{f_{n m}}{\sqrt{\epsilon_{p}\left(f_{n m}^{\prime}\right)}} .
$$

Inserting Eq. (3), we find the mode frequencies of the inductively shunted cavity to be

$$
f_{n m}^{\prime}=\sqrt{f_{n m}^{2}+f_{p}^{2}} .
$$

This expression has both a cutoff frequency, the plasma frequency $f_{p}$, and also has the desired behavior of reducing to Eq. (1) as $r / a \rightarrow 0$. It has previously been used empirically as a fit to FE simulations of cavities containing arrays of thin conducting cylinders [21].

We performed HFSS eigenmode simulations to verify Eq. (6) over a range of $r / a$, shown in Fig. 2, finding good agreement for $r \ll a$. As $r / a$ increases beyond around 0.1, the model breaks down due to increasing Bragg scattering [22]. 


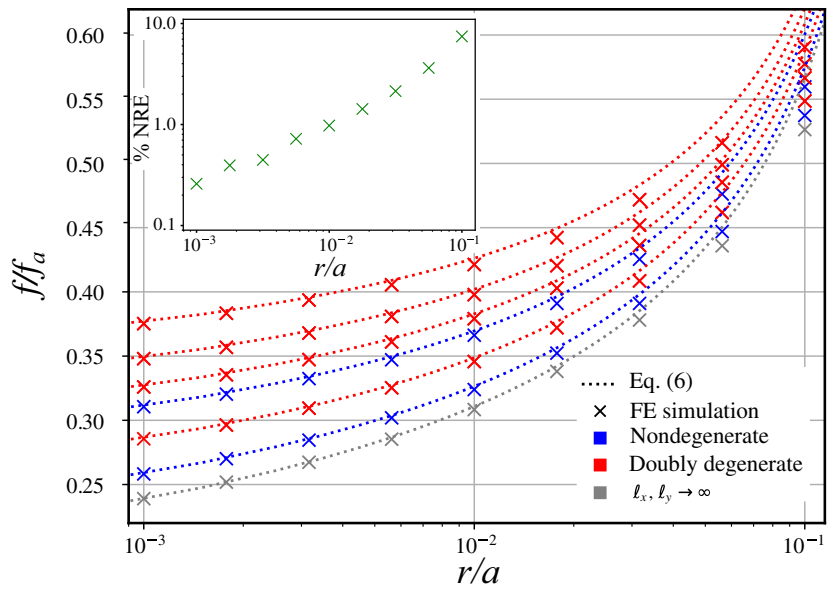

FIG. 2. The lowest ten modes for a cavity containing a containing an inductive shunt array, with $\ell_{x}, \ell_{y}=10 \mathrm{a}$. The mode frequencies and degeneracies are accurately predicted by the plasma model. Also included in gray are results for the fundamental mode when $\ell_{x}, \ell_{y} \rightarrow \infty$, with simulation values found using a method described in Ref. [23]. Inset shows the normalized relative error (NRE) between simulation and Eq. (6) $\Sigma_{i=1}^{10}\left|f_{i_{\mathrm{FE}}}-f_{i_{\mathrm{eq}}}\right| / 10 f_{i_{\mathrm{FE}}}$.

We can use Eq. (6) to find the band structure of the cavity in the limit $\ell_{x}, \ell_{y} \rightarrow \infty$, by substituting the wave numbers $n \pi / \ell_{x} \rightarrow k_{x}, m \pi / \ell_{y} \rightarrow k_{y}$ into Eq. (1). On expanding Eq. (6), we then find the following quadratic mode spectrum near the plasma frequency

$$
f=f_{p}\left(1+\frac{1}{2} k^{2} / k_{p}^{2}\right)
$$

where $k^{2}=k_{x}^{2}+k_{y}^{2}, k_{p}=\sqrt{\epsilon_{0} \epsilon_{r} \mu_{0}} \omega_{p}$, and $\omega_{p}=2 \pi f_{p}$.

\section{Circuit model $(r / a>0.1)$}

In this section, we develop a circuit model for the inductively shunted cavity valid for $r / a>0.1$, where the plasma model has broken down. In this limit, we model the array of shunts as breaking up the cavity into an array of smaller cavities. We take the gaps between the shunts into account by allowing neighboring cavities to magnetically couple to one another.

The tight-binding model has been used to model such coupled-cavity arrays [24], and circuit models have also been used to model one-dimensional coupled-cavity arrays $[25,26]$. Here, we extend the circuit-model treatment to two-dimensional arrays, and verify that for $r / a>0.1$, it provides an accurate model for the inductively shunted cavity.

Figure 3 shows a section of the circuit, from which we construct the impedance matrix $\mathbf{Z}_{\mathbf{2 D}}$ using mesh analysis. This matrix can be mapped exactly into the simpler impedance matrix $\mathbf{Z}_{\mathbf{1 D}}$ of a one-dimensional coupledcavity array (see Appendix B for details). For the three

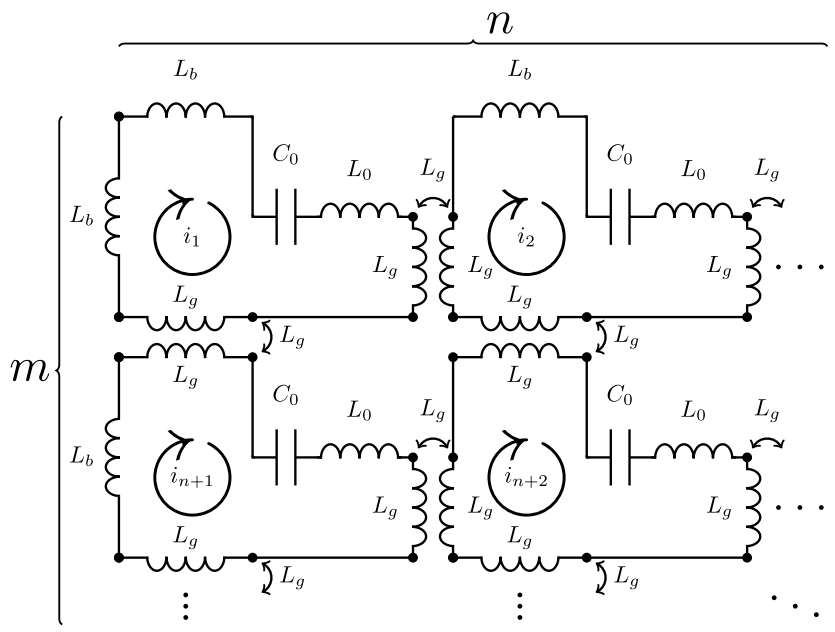

FIG. 3. Circuit representation for the lowest $n \times m$ modes of a $n \times m$ array of nearest-neighbor magnetically coupled cavities formed between $(n-1) \times(m-1)$ inductive shunts. The isolated fundamental mode of each cavity is represented by $L_{0}$ and $C_{0}$. The magnetic coupling between cavities is given by the mutual inductances $L_{g}$. The circuit includes boundary inductances $L_{b}$, that can be used to include asymmetry in the outermost cavities. The mutual inductance coupling coefficient $k$ (where $M=k \sqrt{L_{1} L_{2}}$ ) has been set to unity for simplicity, as differences can be absorbed into $L_{0}$ and $L_{b}$.

specific inductance ratios $L_{b} / L_{g}=0,1,2$ the circuit has simple closed-form solutions. Taking $L_{b}=0$ (corresponding to there being no edge effects for cavities at the border of the array), we find the mode frequencies of the inductively shunted cavity to be

$$
\begin{aligned}
f_{i j} & =\frac{f_{0}}{\sqrt{1+4 \beta\left\{1+\frac{1}{2}\left[\cos \left(\frac{i \pi}{n}\right)+\cos \left(\frac{j \pi}{m}\right)\right]\right\}}}, \\
f_{0} & =1 /\left(2 \pi \sqrt{L_{0} C_{0}}\right) \quad \beta=L_{g} / L_{0} \quad(1 \leq i \leq n, 1 \leq j \leq m),
\end{aligned}
$$

where $f_{0}$ is the frequency of each uncoupled cavity in the circuit model, and $\beta$ is the inductive coupling parameter between nearest-neighbor cavities. Taking $n, m \rightarrow \infty$, we find a new cutoff frequency for the inductively shunted cavity, $f_{c}=f_{0} / \sqrt{1+8 \beta}$.

The field distribution of modes is now significantly altered by the inductive shunt array. We can find the relative field amplitude inside each cavity from the eigenvectors of $\mathbf{Z}_{2 \mathbf{D}}$. For $L_{b}=0$, we find

$$
\begin{aligned}
E_{i j}(a, b)= & E_{0} \sin \left[\frac{i(2 a-1) \pi}{2 n}\right] \sin \left[\frac{j(2 b-1) \pi}{2 m}\right] \\
& (1 \leq a, i \leq n) \quad(1 \leq b, j \leq m),
\end{aligned}
$$

where $E_{i j}$ is the relative electric field amplitude in each cavity, $a$ and $b$ index these cavities, and $i$ and $j$ index the 
modes. The lowest mode $(i, j=1)$ is symmetric, and the highest mode $(i=n, j=m)$ is antisymmetric, as we would expect for hybridized modes.

Note that this circuit model tends to the tight-binding model for $\beta \ll 1$. A series expansion of Eq. (8) in powers of $\beta$ results in

$$
\begin{aligned}
f_{i j} & \approx f_{0}-2 t\left[2+\cos \left(k_{x} a\right)+\cos \left(k_{y} a\right)\right], \\
t & =\beta f_{0} / 2 \quad k_{x}=i \pi / \ell_{x} \quad k_{y}=j \pi / \ell_{y},
\end{aligned}
$$

which is the tight-binding model dispersion for a square lattice. A series expansion of the cosine terms in Eq. (8) instead results in the following quadratic mode spectrum near the cutoff frequency

$$
f=f_{c}\left(1+\frac{1}{2} k^{2} / k_{0}^{2}\right)
$$

where $k^{2}=k_{x}^{2}+k_{y}^{2}, k_{0}^{2}=1 /\left(\beta a^{2}\right)$, and $k_{x}$ and $k_{y}$ are defined in Eq. (10).

In Fig. 4 we show results of HFSS eigenmode simulations of cavities containing inductive shunt arrays with $r / a=0.25$. We find good agreement to our circuit model, which improves further when we include a next-nearestneighbor coupling parameter $\beta_{1}$ (see Appendix $\mathrm{B}$ for details).

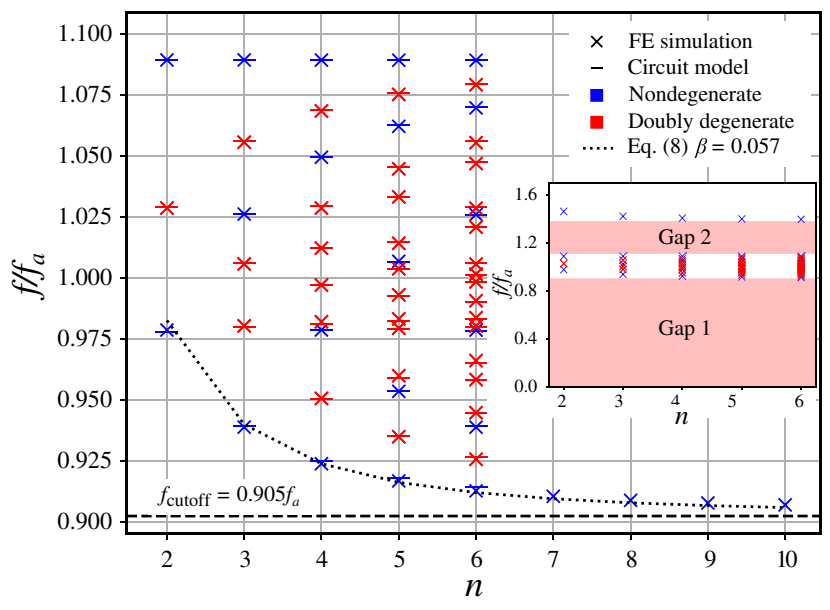

FIG. 4. Lowest $n^{2}$ modes for a cavity with size $\ell_{x}=\ell_{y}=a \times$ $n$, containing $n-1 \times n-1$ inductive shunts, with $r=0.25 a$. For $n$ between 2-6, the fit to the next-nearest-neighbor circuit model is shown for all $n^{2}$ modes, fitted with nearest and nextnearest-neighbor couplings $\beta$ and $\beta_{1}$ as free parameters. For $n$ between 7-10, only the fundamental frequency from FE simulation is shown. Dotted line shows the fundamental frequency decreasing towards a bound in agreement with Eq. (8). Inset shows the lowest $n^{2}+1$ modes, showing two band gaps: below mode 1 and between modes $n^{2}$ and $n^{2}+1$.

\section{INTERQUBIT COUPLING AND DRIVE-LINE CROSS TALK}

We now use the results of Sec. II to predict the form of cavity-mediated cross-talk for superconducting qubits inside enclosures with inductive-shunt arrays. In particular, we consider the transverse exchange coupling $J_{i j}$ between qubits $i$ and $j$, and the drive coupling $\varepsilon_{i j}$ of localized drive line $i$ to qubit $j$. The corresponding Hamiltonian operators are as follows:

$$
\begin{aligned}
& \hat{H}_{i j}^{J}=J_{i j}\left(\hat{a}_{i} \hat{a}_{j}^{\dagger}+\hat{a}_{i}^{\dagger} \hat{a}_{j}\right), \\
& \hat{H}_{i j}^{D}=\varepsilon_{i j}\left(\hat{a}_{j}-\hat{a}_{j}^{\dagger}\right) V_{i} .
\end{aligned}
$$

Here $\hat{a}_{i}^{\dagger}$ and $\hat{a}_{i}$ are the creation and annihilation operators of qubit mode $i, V_{i}$ is the voltage on drive line $i$, and "localized drive line" refers to a drive that interacts with the enclosure from a localized source. In the absence of any shunts, we expect the mechanism of cavity-mediated cross-talk to be through the standing-wave EM modes of the cavity. However, in the presence of the inductive shunt array, no standing waves can form below the cutoff frequency. Instead, the divergence in the density of cavity modes around the cutoff frequency results in a radically different form of cavity-mediated cross-talk.

The plasma model of periodic inductive shunts provides an intuitive framework for predicting interqubit couplings and drive-line cross-talk in this case. In this framework, an excited superconducting qubit or drive line, oscillating below the plasma frequency, sees the cavity instead as a parallel-plate waveguide below cutoff, and drives an evanescent radial waveguide mode into the cavity. We consider only the dominant $\mathrm{TM}_{00}$ radial mode [27] (Fig. 10 in Appendix C). This then results in a transverse coupling strength $J_{i j}$ between transmon qubits $i$ and $j$ with the following form (see Appendix C)

$$
\begin{aligned}
& J_{i j}=-2 g^{2} \frac{\omega_{q}}{\left(|v| / \delta_{0}\right)^{2}} K_{0}\left(d_{i j} / \delta_{p}\right), \\
& \delta_{p}=1 / \sqrt{\epsilon_{0} \epsilon_{r} \mu_{0}\left(\omega_{p}+\omega_{q}\right)\left(\omega_{p}-\omega_{q}\right)},
\end{aligned}
$$

where $d_{i j}$ is the qubit separation, $\delta_{p}$ is the plasma penetration depth, and $g$ is an effective coupling strength between the qubits and the $\mathrm{TM}_{00}$ waveguide mode; $v$ is the speed of light in the waveguide, and $\delta_{0}$ is the interaction length between qubits and the waveguide; $K_{0}$ is a modified Bessel function of the second kind, and we take qubits $i$ and $j$ to have equal frequency. If the source of the excitation is instead drive line $i$, this results in a drive coupling $\varepsilon_{i j}$ to qubit $j$ with the same spatial dependence

$$
\varepsilon_{i j}=\varepsilon_{0} K_{0}\left(d_{i j} / \delta_{p}\right)
$$


where the drive is resonant with qubit $j$. For $d_{i j} \gg \delta_{p}$, these expressions have simple asymptotic forms, since

$$
K_{0}\left(d_{i j} / \delta_{p}\right) \rightarrow \sqrt{\pi / 2} \times e^{-d_{i j} / \delta_{p}} / \sqrt{d_{i j} / \delta_{p}} .
$$

This predicted exponential decay in interqubit coupling and drive-line cross-talk is a very useful property where only local qubit connectivity is desired. Note that we can express the plasma penetration depth as a function of only $\omega_{q}, r$, and $a$

$$
\delta_{p}=a \sqrt{\left[\ln \left(\frac{a}{r}\right)-\Pi\right] / 2 \pi} \sqrt{1 /\left[1-\left(\omega_{q} / \omega_{p}\right)^{2}\right]},
$$

where $\omega_{p}$ is itself a function of $r$ and $a$ given by Eq. (4), and where $\Pi$ is also defined in Eq. (4).

We can instead derive the cavity-mediated coupling between qubits by considering the interaction of qubits with the new distribution of cavity modes in the inductively shunted cavity. From Eqs. (7) and (11), we see that the inductively shunted cavity is characterized by a 2D quadratic mode spectrum above the cutoff frequency. A qubit with a frequency below the cutoff interacts with these modes to form a bound state [28], with a spatially exponentially decaying envelope. These bound states then mediate a coupling between distant qubits [29]. Remarkably, for equal frequency qubits below the cutoff of a 2D quadratic mode spectrum, the predicted bound-statemediated transverse coupling $[29,30]$ has exactly the same spatial dependence as Eq. (14), with the plasma penetration depth replaced by the bound-state length

$$
\delta_{b}=\sqrt{\alpha \omega_{b} /\left(\omega_{b}-\omega_{q}\right)},
$$

where $\alpha$ characterizes the curvature of the band edge, given by $\omega=\omega_{b}\left[1+\alpha\left(k-k_{0}\right)^{2}\right]$. We can find $\alpha$ from Eq. (7) and compare the predicted bound-state length with the plasma penetration depth in Eq. (15). We find

$$
\delta_{b}=1 / \sqrt{\epsilon_{0} \epsilon_{r} \mu_{0} 2 \omega_{p}\left(\omega_{p}-\omega_{q}\right)}
$$

This result holds for qubits close in frequency to the band edge, where the quadratic dispersion approximation is valid; in which case $\omega_{q} \approx \omega_{p}$ and $\delta_{b} \approx \delta_{p}$. Thus the spatial dependence of qubit coupling for these two seemingly disparate models agrees well. Finding $\alpha$ instead from Eq. (11), we find

$$
\delta_{b}=a \sqrt{\beta / 2} \sqrt{1 /\left(1-\omega_{q} / \omega_{b}\right)} .
$$

Note that this last result applies not just to inductively shunted cavities, but more generally to $2 \mathrm{D}$ coupled-cavity arrays.

\section{FE SIMULATION OF MONOLITHIC SUPERCONDUCTING QUBIT DEVICE}

We now move on to perform FE simulations of a realistic superconducting circuit device, and compare the results with the predictions of the previous sections. We perform HFSS simulations on the superconducting qubit device model shown in Fig. 5. This model is based on an architecture in which universal quantum control and readout have been demonstrated [31,32]. For the purpose of probing cavity-mediated cross-talk qubit couplers and readout circuitry are not included in the model, although we illustrate how a nearest-neighbor capacitive-coupling network might be implemented in Fig. 5(c).

The model consists of a $21 \times 21$ array of coaxial transmon qubit islands on a silicon $\left(\epsilon_{r}=11.9\right)$ substrate measuring $\ell_{x}=42 \mathrm{~mm}, \ell_{y}=42 \mathrm{~mm}, \ell_{z}=0.5 \mathrm{~mm}$, enclosed by a perfectly conducting cavity, which is inductively shunted by a $20 \times 20$ array of perfectly conducting cylinders. The qubit islands and cylinders are spaced by $a=$ $2 \mathrm{~mm}$, and each pair of qubit islands is capacitively coupled to an off-chip coaxial drive line. The qubits have $C_{J} \approx$ $100 \mathrm{fF}$ and $L_{J} \approx 10 \mathrm{nH}$ at $f_{01}=5 \mathrm{GHz}$, corresponding to $E_{J} / E_{C} \approx 80$.

The fundamental mode frequency of the cavity over a range of cylinder radii is summarized in Table I. In the absence of any cylinders, the fundamental frequency of the enclosure is well below the typical range of transmon frequencies, which results in a high density of enclosure modes around qubit frequencies. However, in the presence of the considered shunting arrays, the fundamental mode frequency is in all cases greater than $11 \mathrm{GHz}$. For $r / a>0.1$, Eq. (6) rapidly diverges from the simulation result as it breaks down due to Bragg scattering. For $r / a<$ 0.1 , we attribute the difference to the vacuum regions in the simulation model introduced by the drive lines, which are not included in Eq. (6). Removal of the qubit pads results in small $(<1 \%)$ changes to simulated cavity-mode frequencies.

We define a reference qubit (drive) $i=0$ at the center of the array, and simulate the transverse coupling (driveline cross-talk) to qubit $j=1,2, \ldots, 10$ using a simple impedance formula [33]. While simulating these properties between qubit (drive) $i$ and qubit $j$, the Josephson junctions in all other qubits are replaced by open circuits [33]. We re-express our results using

$$
\begin{aligned}
& \Gamma_{0, j}^{Q}=J_{0, j} / J_{0,1}, \\
& \Gamma_{0, j}^{D}=\varepsilon_{0, j} / \varepsilon_{0,1} .
\end{aligned}
$$

These expressions eliminate the unknown prefactors in Eqs. (14) and (16) considering qubits, which couple equally to the evanescent waveguide mode. In this case $\Gamma^{Q}=\Gamma^{D}$, since both are then only a measure of the spatial decay of the waveguide mode. Ignoring the 

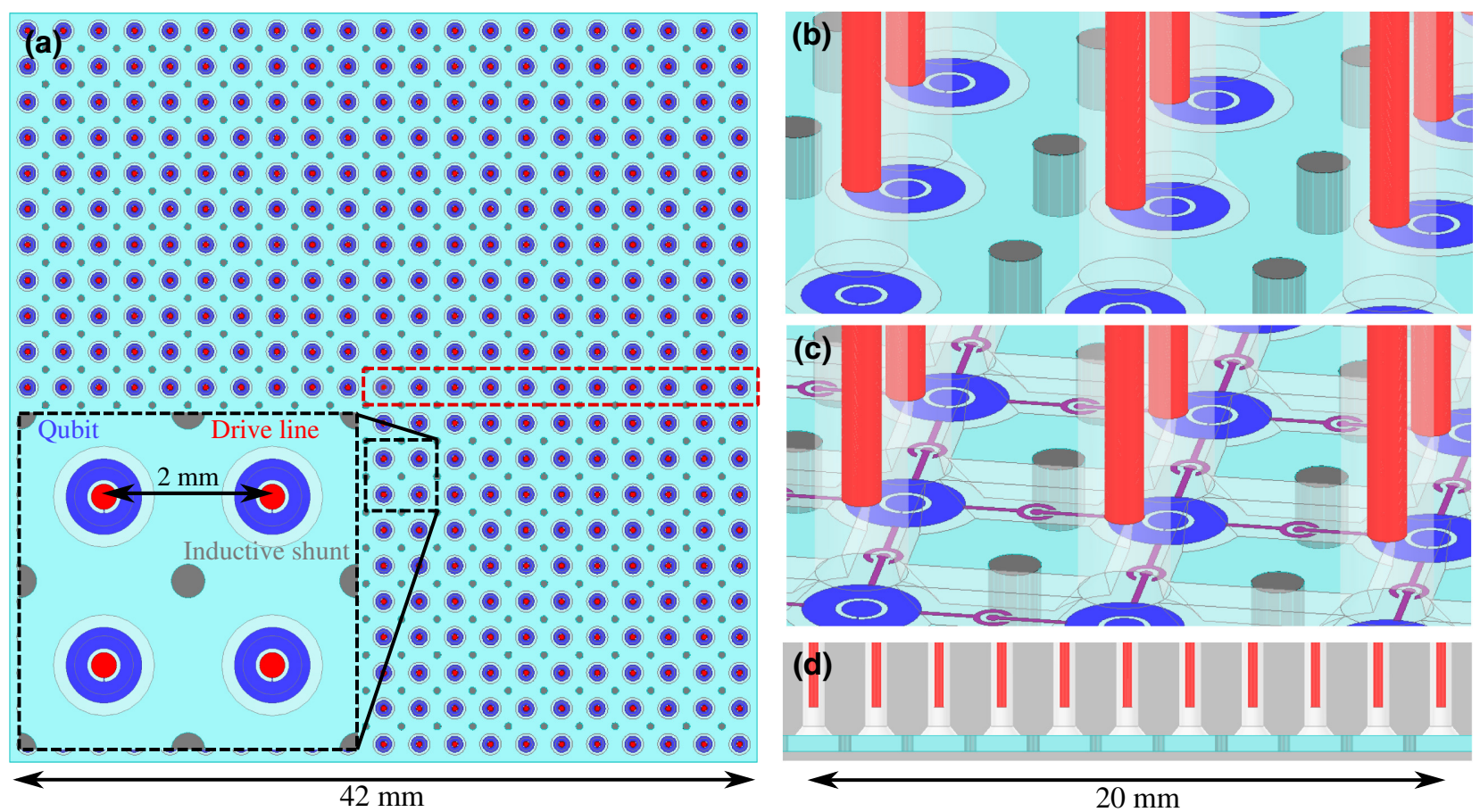

FIG. 5. (a) Top-down view of the model. The qubits for which $J$ and drive-line cross-talk are simulated are indicated by the dashed red line. (b) View showing the drive lines (red) above each qubit, and the inductive shunts (gray, here shown with $r=0.2 \mathrm{~mm}$ ) passing through the substrate. (c) Illustrating how the design can be modified to include a nearest-neighbor direct capacitive-coupling network (purple) between qubits. (d) Cross section of the dashed-red region in (a). The model is enclosed by a perfect conductor (shown in gray), representing an ideal superconducting enclosure.

weak square-root dependence on qubit separation, these expressions approximate to

$$
\Gamma_{0, j}^{Q}=\Gamma_{0, j}^{D} \approx e^{-d_{1, j} / \delta_{p}}
$$

We find good agreement between simulation and the plasma model of interqubit coupling and drive-line crosstalk for a range of qubit frequencies and shunt radii (see Fig. 6). When calculating the plasma penetration depth [Eq. (15)], we use the fundamental cavity frequency found from eigenmode simulation (Table I) as the plasma frequency, rather than that from Eq. (4). With this choice, we find that the plasma model of interqubit coupling still agrees well with simulation for $r / a>0.1$.

The plasma penetration depth is of the order of the shunt separation, and decreases significantly with increasing shunt radius. For qubits well below the plasma frequency, it has only a weak dependence on the qubit frequency.
Note that although we use coaxial qubits in our simulation, we can expect the qubit geometry only to affect the coupling $g$ to the dominant $\mathrm{TM}_{00}$ waveguide mode, and therefore expect good agreement with the model for other qubit geometries.

Finally, any real quantum processor requires coupling circuitry between qubits to facilitate entangling gates. This circuitry only slightly perturbs the mediating $\mathrm{TM}_{00}$ waveguide mode because it lies perpendicular to the electric field of this mode, and so cavity-mediated cross-talk is not altered significantly. However, such circuitry introduces circuit-mediated cross-talk channels, the study of which is beyond the scope of this work. We do note that in an idealized lumpedelement model of a 2D grid of nearest-neighbor capacitively coupled qubits, circuit mediated crosstalk decays exponentially with spatial separation (see Appendix D).

TABLE I. Fundamental frequency of the enclosure in Fig. $5(\mathrm{GHz})$, changing the inductive shunt radius (mm).

\begin{tabular}{lccccccccc}
\hline \hline & $0^{\mathrm{a}}$ & 0.05 & 0.1 & 0.15 & 0.2 & 0.25 & 0.3 & 0.35 & 0.4 \\
\hline FE $^{\mathrm{b}}$ & 1.49 & 11.89 & 13.95 & 15.78 & 17.58 & 19.42 & 21.37 & 23.44 & 25.14 \\
Eq. (6) & 1.46 & 11.34 & 13.43 & 15.39 & 17.47 & 19.82 & 22.68 & 26.40 & 31.74 \\
\hline \hline
\end{tabular}

${ }^{a}$ No inductive shunts.

${ }^{\mathrm{b}}$ HFSS finite-element eigenmode simulation. 
(a)

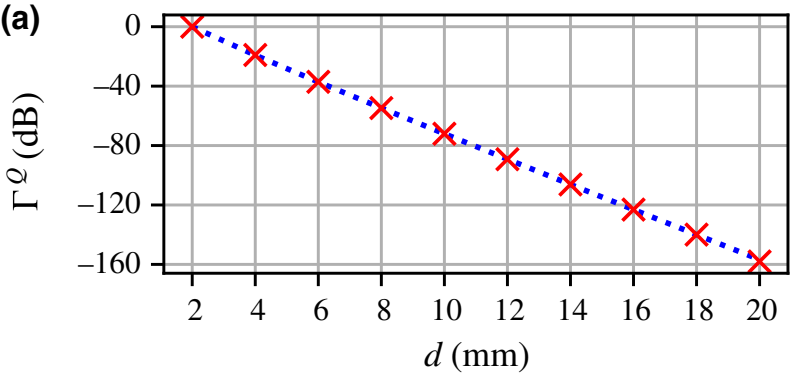

(b)

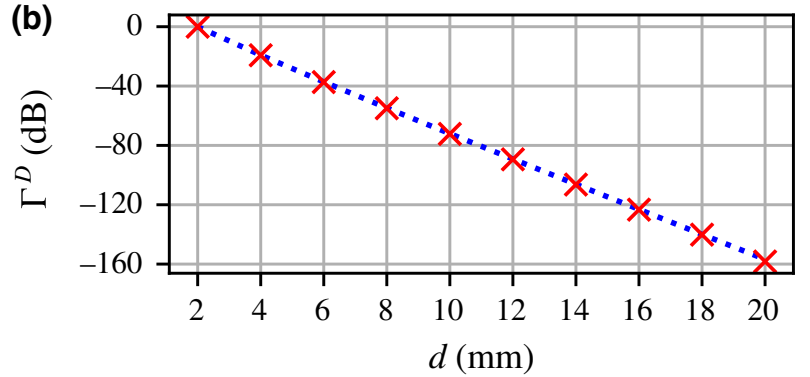

(c)

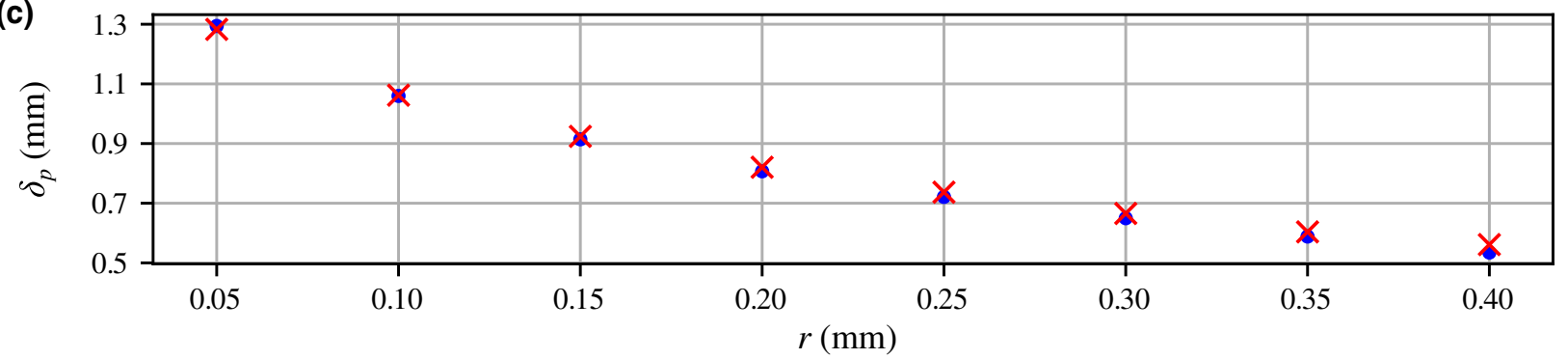

(d)

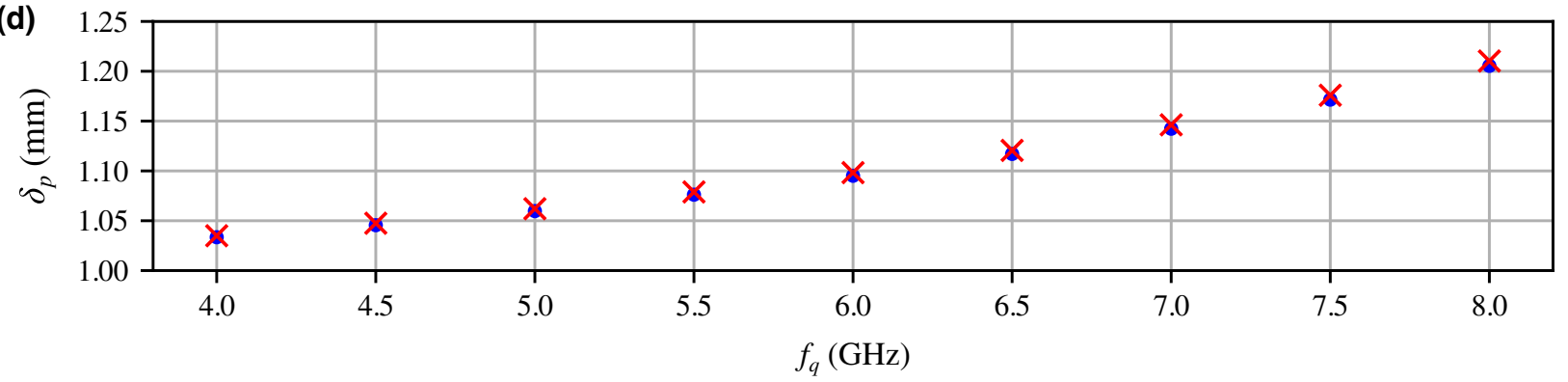

FIG. 6. (a),(b) Interqubit and drive line to qubit cross-talk for $r=0.1 \mathrm{~mm}, f_{q}=5 \mathrm{GHz}$. Red crosses are FE simulation values, and blue dotted lines are the prediction of Eqs. (14)-(16). (c),(d) Plasma penetration depth against shunt radius and qubit frequency. Red crosses are calculated using Eq. (15) and blue dots are found by fitting Eq. (14) to the FE simulation $\Gamma^{Q}$ values, with $\delta_{p}$ as the sole fit parameter. In (c) $f_{q}=5 \mathrm{GHz}$, and in (d) $r=0.1 \mathrm{~mm}$.

\section{CONCLUSIONS}

We first develop a plasma and circuit model to accurately predict the mode frequencies of enclosures inductively shunted by periodic perfectly conducting cylinder arrays. We then use these models to predict the exponential decay of cavity-mediated interqubit coupling and drive-line cross-talk for superconducting circuits inside such enclosures. The plasma model, in particular, predicts the fundamental enclosure frequency and the rate of cross-talk decay to be simple functions of the shunt radius and spacing [Eqs. (6) and (18)], providing a tool for the design of the shunt array. The predictions agree well with a FE simulation of a realistic device. These results indicate that monolithic superconducting circuit architectures that employ inductive shunt arrays can scale arbitrarily in size, with enclosure-mediated cross-talk that is small and local in nature, making this a promising approach for quantum computation with superconducting circuits. An interesting direction for further work is to provide models for the cross-talk arising from different networks of qubit-coupling circuitry.

\section{ACKNOWLEDGMENTS}

P.S. acknowledges support from NQIT (Networked Quantum Information Technologies). T.T. acknowledges support from the Masason foundation and the Nakajima Foundation. B.V. acknowledges support from an EU Marie Skodowska-Curie fellowship. P.L. acknowledges support from the EPSRC (EP/M013243/1) and Oxford Quantum Circuits Limited. We thank Conal Murray and Mustafa Bakr for insightful discussions, and Sophia Sosnina for technical contributions.

\section{APPENDIX A: EFFECT OF MULTIPLE DIELECTRIC LAYERS}

Here we consider replacing the single dielectric in the cavity with multiple layers of dielectric as in Fig. 7. A relevant case for superconducting quantum circuits is that with three layers: vacuum, substrate, vacuum.

Since the magnetic properties of the cavity are unaltered, the introduction of multiple dielectric layers only affect the capacitance of the $l=0$ modes, which take the form of a 


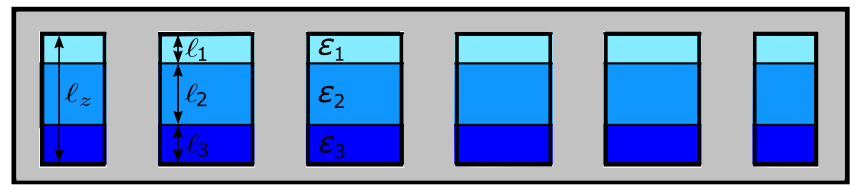

FIG. 7. Cross section of the inductively shunted cavity, now containing a stack of different dielectric materials.

parallel-plate capacitance between the top and bottom of the cavity

$$
C=k \times \frac{\epsilon_{r}}{\ell_{z}}
$$

where $k$ is a constant. The capacitance in the presence of multiple dielectric layers $C^{\prime}$ is the series sum of the parallel-plate capacitances across each layer

$$
\begin{gathered}
C^{\prime}=k \times 1 / \Sigma_{i=1}^{n}\left(\frac{\ell_{i}}{\epsilon_{i}}\right)=k \times \frac{\epsilon_{r}^{\prime}}{\ell_{z}}, \\
\epsilon_{r}^{\prime}=\ell_{z} / \Sigma_{i=1}^{n}\left(\frac{\ell_{i}}{\epsilon_{i}}\right) .
\end{gathered}
$$

Thus the only effect of a dielectric stack is to replace the relative permitivitty $\epsilon_{r}$ wherever it appears, with the effective relative permitivitty $\epsilon_{r}^{\prime}$ in Eq. (A3).

\section{APPENDIX B: MESH ANALYSIS AND MAPPING THE ARRAY CIRCUIT TO THE CHAIN CIRCUIT}

Using mesh analysis [34], the circuit in Fig. 3 of the main text can be represented by

$$
\mathbf{Z}_{\mathbf{2 D}}\left[\begin{array}{c}
i_{1} \\
\vdots \\
i_{i} \\
\vdots \\
i_{n m}
\end{array}\right]=\left[\begin{array}{c}
V_{1} \\
\vdots \\
V_{i} \\
\vdots \\
V_{n m}
\end{array}\right]
$$

where $\mathbf{Z}_{\mathbf{2 D}}$ is an $n m \times n m$ square matrix, $i_{i}$ is the current through mesh $i$ and $V_{i}$ is the voltage applied to mesh $i$. At the mode frequencies, currents can oscillate in the absence of any excitations $\left(V_{i}=0\right)$, therefore modes exist at frequencies where an eigenvalue of $\mathbf{Z}_{\mathbf{2 D}}$ is 0 .

$\mathbf{Z}_{2 D}$ can be expressed as a diagonal block matrix. For a nearest-neighbor coupling model this block matrix is tridiagonal, while for a next-nearest-neighbor coupling model it is pentadiagonal, and so on. We consider the cases of nearest-neighbor and next-nearest-neighbor couplings. Expressed in block form, $\mathbf{Z}_{\mathbf{2 D}}$ is a $m \times m$ matrix of $n \times n$ matricies. For nearest-neighbor coupling, it takes the form

$$
\mathbf{Z}_{\mathbf{2} \mathbf{D}_{\mathbf{m} \times \mathbf{m}}}=\left[\begin{array}{ccccccc}
\mathbf{Z}_{\alpha} & \mathbf{Z}_{\mathbf{G}} & & & \cdots & & \mathbf{0} \\
\mathbf{Z}_{\mathbf{G}} & \mathbf{Z} \beta & \mathbf{Z}_{\mathbf{G}} & & & & \\
& \mathbf{Z}_{\mathbf{G}} & \mathbf{Z} \beta & \mathbf{Z}_{\mathbf{G}} & & & \\
& & \ddots & \ddots & \ddots & & \\
& & & \mathbf{Z}_{\mathbf{G}} & \mathbf{Z} \beta & \mathbf{Z}_{\mathbf{G}} & \\
& & & & \mathbf{Z}_{\mathbf{G}} & \mathbf{Z} \beta & \mathbf{Z}_{\mathbf{G}} \\
\mathbf{0} & & \cdots & & & \mathbf{Z}_{\mathbf{G}} & \mathbf{Z} \alpha
\end{array}\right]
$$

where

$$
\begin{aligned}
\mathbf{Z} \alpha_{\mathbf{n} \times \mathbf{n}} & =\left[\begin{array}{ccccccc}
Z_{\alpha_{1}} & -Z_{g} & & & \cdots & & 0 \\
-Z_{g} & Z_{\alpha_{2}} & Z_{b} & & & & \\
& -Z_{g} & Z_{\alpha_{2}} & -Z_{g} & & & \\
& & \ddots & \ddots & \ddots & & \\
& & & -Z_{g} & Z_{\alpha_{2}} & -Z_{g} & \\
0 & & \ldots & & -Z_{g} & Z_{\alpha_{2}} & -Z_{g} \\
Z_{\alpha_{1}} & =Z_{0}+2 Z_{g}+2 Z_{b} & & & & Z_{g} & Z_{\alpha_{1}}
\end{array}\right] \\
Z_{\alpha_{2}} & =Z_{0}+3 Z_{g}+Z_{b}
\end{aligned}
$$

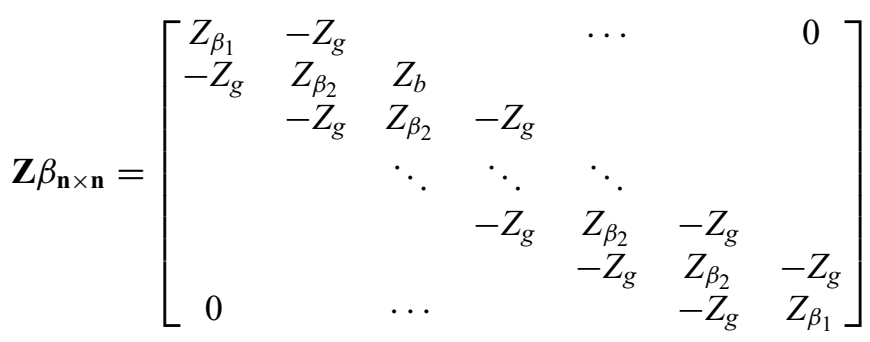

$$
\begin{aligned}
& Z_{\beta_{1}}=Z_{0}+3 Z_{g}+Z_{b} \\
& Z_{\beta_{2}}=Z_{0}+4 Z_{g}
\end{aligned}
$$$$
\mathbf{Z}_{\mathbf{G}_{\mathbf{n} \times \mathbf{n}}}=-Z_{g} \mathbf{I}_{\mathbf{n} \times \mathbf{n}}
$$

and

$$
Z_{0}=i \omega L_{0}-\frac{i}{\omega C_{0}} \quad Z_{g}=i \omega L_{g} \quad Z_{b}=i \omega L_{b}
$$

. It can be verified by expansion that $\mathbf{Z}_{\mathbf{2}}$ can be written

$$
\mathbf{Z}_{\mathbf{2} \mathbf{D}_{\mathbf{n m} \times \mathbf{n m}}}=\mathbf{Z}_{\mathbf{1} \mathbf{D}_{\mathbf{n} \times \mathbf{n}}} \oplus \mathbf{Z}_{\mathbf{1} \mathbf{D}_{\mathbf{m} \times \mathbf{m}}}-Z_{0} \mathbf{I}_{\mathbf{n m} \times \mathbf{n m}},
$$

where $\oplus$ is the Kronecker sum and

$$
\begin{aligned}
\mathbf{Z}_{\mathbf{1} \mathbf{\mathbf { D } _ { \mathbf { n } } \mathbf { n }}} & =\left[\begin{array}{ccccc}
Z_{1} & -Z_{g} & & \cdots & 0 \\
-Z_{g} & Z_{2} & -Z_{g} & & \\
& \ddots & \ddots & \ddots & \\
& & -Z_{g} & Z_{2} & -Z_{g} \\
0 & \cdots & & -Z_{g} & Z_{1}
\end{array}\right] \\
Z_{1} & =Z_{0}+Z_{g}+Z_{b} \\
Z_{2} & =Z_{0}+2 Z_{g}
\end{aligned}
$$

$\mathbf{Z}_{\mathbf{1} \mathbf{D}_{\mathbf{n} \times \mathbf{n}}}$ is exactly the impedance matrix of the circuit in Fig. 8. This mapping makes solving the eigenvalues of $\mathbf{Z}_{\mathbf{2 D}}$ 


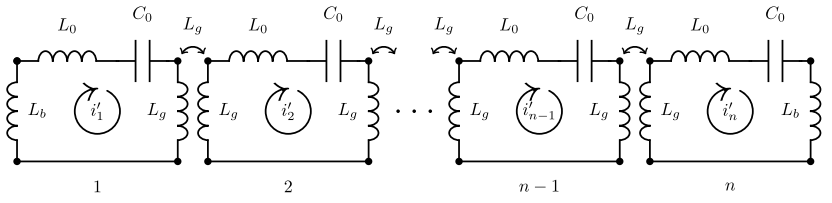

FIG. 8. Circuit representation for the lowest $n$ modes of a chain of $n$ magnetically coupled cavities.

much easier, since

$$
\lambda\left(\mathbf{Z}_{\mathbf{2 D}}\right)_{i j}=\lambda\left(\mathbf{Z}_{\mathbf{1 D}}\right)_{i}+\lambda\left(\mathbf{Z}_{\mathbf{1 D}}\right)_{j}-Z_{0} .
$$

Therefore, finding the mode frequencies of the $2 \mathrm{D}$ circuit is reduced to the problem of solving the eigenvalues of the 1D circuit, inserting these into Eq. (B3), and solving for $\lambda\left(\mathbf{Z}_{\mathbf{2 D}}\right)_{i j}=0$.

$\mathbf{Z}_{1 \mathbf{D}}$ is a tridiagonal matrix that is also almost a toeplitz matrix except for the elements $\mathbf{Z}_{\mathbf{1 D} 11}$ and $\mathbf{Z}_{\mathbf{1 D} n n}$. Simple closed-form solutions for this type of matrix are known to exist for particular values of these boundary elements [35], in our case when $L_{b}=0, L_{g}, 2 L_{g}$. Using these solutions and substituting into Eq. (B3) leads to the following mode frequencies for the circuit in Fig. 3 of the main text

$$
\begin{aligned}
f_{i j} & =\frac{f_{0}}{\left.\sqrt{1+4 \beta\left(1+\frac{1}{2} \gamma_{i j}\right.}\right)}, \\
\gamma_{i j} & =\cos \left(\frac{i \pi}{n}\right)+\cos \left(\frac{j \pi}{m}\right) \quad\left(L_{b}=0\right), \\
\gamma_{i j} & =\cos \left(\frac{j \pi}{n+1}\right)+\cos \left(\frac{j \pi}{m+1}\right) \quad\left(L_{b}=L_{g}\right), \\
\gamma_{i j} & =\cos \left(\frac{(i-1) \pi}{n}\right)+\cos \left(\frac{(j-1) \pi}{m}\right) \quad\left(L_{b}=2 L_{g}\right) .
\end{aligned}
$$

Increasing the boundary inductance $L_{b}$ lowers the frequency of all modes in these solutions.

We find it interesting that the 2D circuit can be mapped directly into the far simpler 1D circuit. We note that this is only true for the special case of identical inductances and capacitances across all unit cells, and that in general, this mapping is not possible.

We now consider the next-nearest-neighbor coupling case, to demonstrate the effect of including further couplings. In this case, it can again be verified by expansion that $\mathbf{Z}_{2 \mathbf{D}}$ can be written in the same form as Eq. (B2), where now

$$
\begin{aligned}
& \mathbf{Z}_{\mathbf{1} \mathbf{D}_{\mathbf{n} \times \mathbf{n}}}=\left[\begin{array}{ccccccc}
Z_{1} & -Z_{g} & -Z_{g_{2}} & & \cdots & & 0 \\
-Z_{g} & Z_{2} & -Z_{g} & -Z_{g_{2}} & & & \\
-Z_{g_{2}} & -Z_{g} & Z_{3} & -Z_{g} & -Z_{g_{2}} & & \\
& & \ddots & \ddots & \ddots & & \\
& & & & & & \\
& & -Z_{g_{2}} & -Z_{g} & Z_{3} & -Z_{g} & -Z_{g_{2}} \\
& & & -Z_{g_{2}} & -Z_{g} & Z_{2} & -Z_{g} \\
0 & & \cdots & & -Z_{g_{2}} & -Z_{g} & Z_{1}
\end{array}\right] \\
& Z_{1}=Z_{0}+Z_{g}+Z_{g_{2}}+Z_{b} \\
& Z_{2}=Z_{0}+2 Z_{g}+Z_{g_{2}} \\
& Z_{3}=Z_{0}+2 Z_{g}+2 Z_{g_{2}} \text {. }
\end{aligned}
$$

This is again the impedance matrix of the circuit in Fig. 8, now with next-nearest-neighbor couplings switched on. Simple closed-form solutions to $\mathbf{Z}_{\mathbf{1}_{\mathbf{n} \times \mathbf{n}}}$ no longer exist in this case, however we still greatly reduce the problem from one of finding the eigenvalues of a $\mathbf{m n} \times \mathbf{m n}$ matrix to one of finding the eigenvalues of an $\mathbf{m} \times \mathbf{m}$ matrix and an $\mathbf{n} \times \mathbf{n}$ matrix.

The agreement of the nearest and next-nearest-neighbor circuit models, as well as the tight-binding model in Eq. (10), to a FE simulation are shown in Fig. 9. The agreement for all models increases as $r / a$ increases. The circuit model offers a better fit than the tight-binding model for a single free parameter.

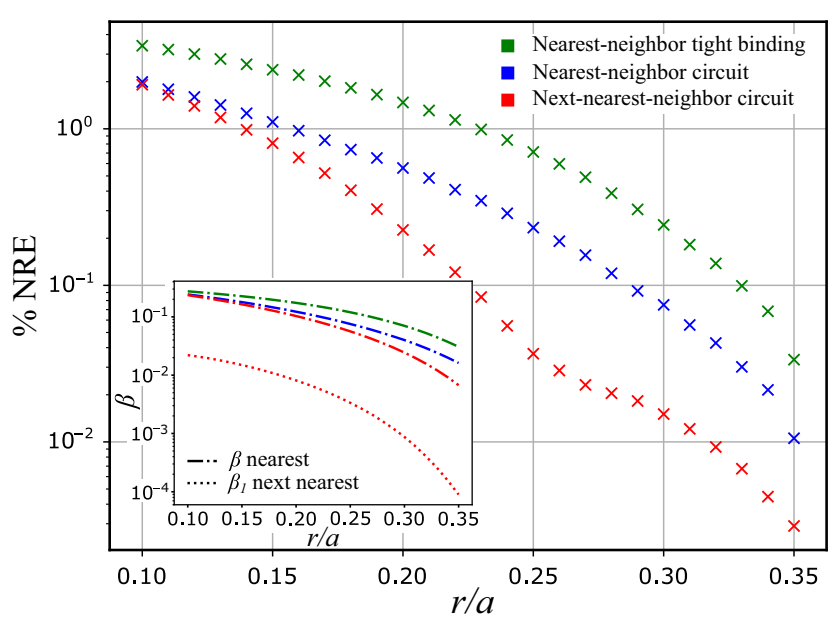

FIG. 9. Normalized relative error $\sum_{i=1}^{16}\left|f_{i_{\mathrm{FE}}}-f_{i_{\text {model }}}\right| / 16 f_{i_{\mathrm{FE}}}$, between a FE simulation and three different fit models, for the lowest 16 modes of a cavity containing a $3 \times 3$ inductive shunt array, changing shunt radius $r$. Inset shows the fitted coupling parameters decreasing smoothly with increasing inductive shunt radius. 


\section{APPENDIX C: TRANSVERSE COUPLING IN PLASMA MODEL}

To find the transverse coupling between the two qubits, we use the impedance formula presented in Ref. [33]

$$
J_{i j}=-\frac{1}{4} \sqrt{\frac{\omega_{i} \omega_{j}}{L_{i} L_{j}}} \operatorname{Im}\left[\frac{Z_{i j}\left(\omega_{i}\right)}{\omega_{i}}+\frac{Z_{i j}\left(\omega_{j}\right)}{\omega_{j}}\right] .
$$

This expression, valid for weakly anharmonic superconducting qubits such as transmon qubits, reduces the problem of finding $J_{i j}$ to that of finding the transimpedance $Z_{i j}(\omega)$ between ports $i$ and $j$, which replace the Josephson junctions of qubits $i$ and $j . \omega_{i}$ is the frequency of qubit $i$, and $L_{i}$ is closely related to the bare junction inductance of qubit $i$ [33].

To find the transimpedance between qubits inside the inductively shunted cavity, we use the circuit model in Fig. 11. Two transmon qubits, with their Josephson junctions replaced by ports, are each capacitively coupled to a parallel-plate waveguide, with $\delta_{0}$ representing the interaction length between the qubits and the waveguide. A current is driven through the port of qubit 1 , which drives an evanescent radial waveguide mode, centered around qubit 1 , into the waveguide. This mode propagates out to qubit 2 , a distance $d_{12}$ away, where it induces a voltage across port 2. The expression for the incident voltage wave along the waveguide for the dominant $\mathrm{TM}_{00}$ mode (depicted in Fig. 10) is [27]

$$
V_{i}(d)=a \times V_{0} H_{0}^{(2)}(k d)
$$

$k$ is the wave number of the line, $a$ is a normalization factor $a=1 / H_{0}^{(2)}\left(k \delta_{0}\right)$, and $H_{0}^{(2)}$ is the Hankel function of the second kind. The current wave has a similar form.

The wave number is given by

$$
k=\sqrt{\mu \epsilon_{0} \epsilon_{r} \epsilon_{p}} \omega
$$

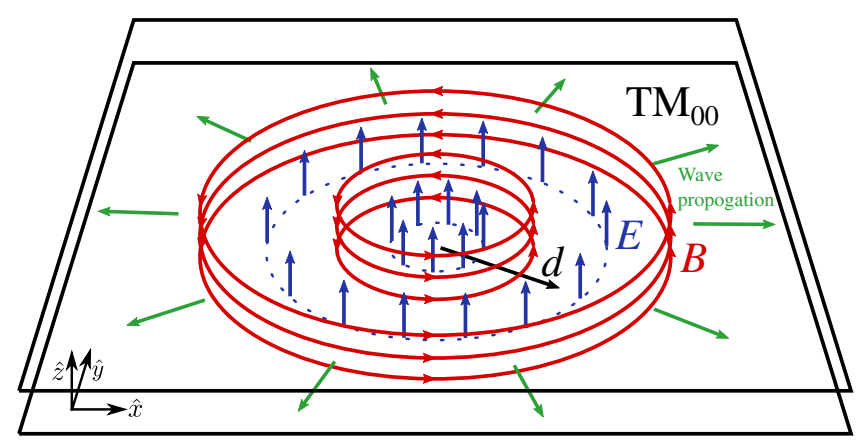

FIG. 10. Visualization of the outward traveling radial $\mathrm{TM}_{00}$ mode of a parallel-plate waveguide, having $E \propto H_{0}^{(2)}(k d), B \propto$ $H_{1}^{(2)}(k d)$.

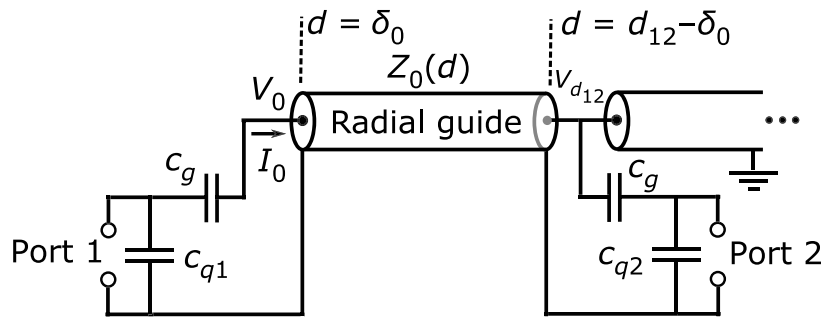

FIG. 11. Circuit model for two transmon qubits (with junctions replaced by ports) separated by distance $d_{12}$ and coupled by the evanescent $\mathrm{TM}_{00}$ mode of a radial waveguide.

where $\epsilon_{p}$ is given by Eq. (3) in the main text, and we use that the electric field is parallel to $\hat{\mathbf{z}}$ for the $\mathrm{TM}_{00}$ mode. $k$ is therefore imaginary at frequencies below the plasma frequency, and the voltage and current waves through the waveguide are evanescent. An important consequence is the characteristic impedance of the waveguide $Z_{0}$ is imaginary in this case.

Substituting our expression for $k$ into Eq. (C2) results in an evanescent voltage along the radial guide

$$
\begin{aligned}
V(d) & =a \times V_{0} H_{0}^{(2)}\left(-i d / \delta_{p}\right), \\
& =b \times V_{0} K_{0}\left(d / \delta_{p}\right),
\end{aligned}
$$

where $\delta_{p}$ is the plasma penetration depth given in Eq. (15) in the main text, $b=1 / K_{0}\left(\delta_{0} / \delta_{p}\right)$, and $K_{0}$ is a modified Bessel function of the second kind. For qubits weakly coupled to the waveguide mode, reflections of the evanescent wave off qubit 2 back at qubit 1 are small, and so we ignore the reflected voltage and current wave. The voltages in the waveguide at the positions of qubit 1 and qubit 2 are then

$$
\begin{gathered}
V\left(\delta_{0}\right)=V_{0}, \\
V\left(d_{12}-\delta_{0}\right)=b \times V_{0} K_{0}\left(d / \delta_{p}\right) .
\end{gathered}
$$

The small magnitude of the reflected voltage and current at $d=\delta_{0}$ means the input impedance of the waveguide is just $Z_{0}\left(\delta_{0}\right)$ (note that $Z_{0}$ is a function of $d$ ). Putting this together, we arrive at

$$
\begin{gathered}
V_{d_{12}} / V_{0}=b \times K_{0}\left(d / \delta_{p}\right), \\
V_{0} / I_{0}=Z_{0}\left(\delta_{0}\right) .
\end{gathered}
$$

We are now equipped to solve the circuit for $Z_{12}$. We define $Z_{g}=1 / i \omega C_{g}, Z_{q 1}=1 / i \omega C_{q 1}, Z_{q 2}=1 / i \omega C_{q 2}$, arriving at

$$
Z_{12}=\frac{Z_{q 1} Z_{q 2} Z_{0}\left(\delta_{0}\right)}{\left(Z_{g}+Z_{q 2}\right)\left[Z_{g}+Z_{0}\left(\delta_{0}\right)\right]} \times b K_{0}\left(d / \delta_{p}\right)
$$

where we use $I_{q 1} \approx V_{q 1} / Z_{q 1}, I_{q 1}$ being the current applied through port 1 . Since $Z_{0}$ is imaginary, we see $Z_{12}$ 
is also imaginary. For large coupling impedance $Z_{g} \gg$ $Z_{q 2}, Z_{0}\left(\delta_{0}\right)$, Eq. (C10) simplifies to

$$
Z_{12}=\frac{Z_{q 1} Z_{q 2} Z_{0}\left(\delta_{0}\right)}{Z_{g}^{2}} \times b K_{0}\left(d / \delta_{p}\right)
$$

We use the following expression for the coupling strength between qubits and the waveguide mode

$$
g=\frac{C_{g}^{\prime}}{2} \sqrt{\frac{\omega_{q} v}{C_{q} C_{r}^{\prime}\left(\delta_{0}\right)}}=\frac{C_{g}^{\prime}}{2} \sqrt{\frac{\omega_{q} Z_{0}\left(\delta_{0}\right)}{C_{q}}} v
$$

where $v$ is the speed of light in the waveguide, $C_{r}^{\prime}\left(\delta_{0}\right)$ is the capacitance per unit length of the waveguide mode at $\delta_{0}$, and $C_{g}^{\prime}$ is the coupling capacitance per unit length between the qubit and the waveguide mode, where $C_{g}=C_{g}^{\prime} \delta_{0}$. Substituting Eqs. (C11) and (C12) into Eq. (C1), we find

$$
J_{i j}=-2 \operatorname{Im}\left[g^{2}\right] \frac{\omega_{q}}{\left(v / \delta_{0}\right)^{2}} b K_{0}\left(d_{12} / \delta_{p}\right)
$$

where $g^{2}$ and $v$ are both negative imaginary. Finally, we redefine $g \rightarrow|g| \times\left[K_{0}\left(\delta_{0} / \delta_{p}\right)\right]^{1 / 2}$ to arrive at Eq. (14) in the main text.

\section{APPENDIX D: CIRCUIT-MEDIATED CROSS-TALK}

An idealized circuit model for a 2D grid of identical transmon qubits with nearest-neighbor capacitive couplings $C_{g}$ is shown in Fig. 12(a). Each node is shunted to ground by a qubit shunt capacitance $C_{q}$ in parallel with a nonlinear Josephson junction [Fig. 12(b)]. To approximate the transverse coupling between qubits $i$ and $j$ at nodes $(0,0)$ and $(n, m)$, respectively, we again use the simple impedance formula presented in Ref. [33]. The first step is to linearize the circuit by replacing all Josephson junctions with ports [Fig. 12(c)]. The transimpedance $Z_{i j}(\omega)$ between the ports at node $(0,0)$ and node $(n, m)$ is then

$$
Z_{i j}(\omega)=\frac{V(n, m)}{V_{0}} Z_{q}
$$

where $Z_{q}=1 / i \omega C_{q}$, and we use $I_{0} \approx V_{0} Z_{q}$, which is valid for $C_{q} \gg C_{g}$. We observe that the linearized circuit in Fig. 12(a) is just a special case of a discretized 2D transmission line circuit model [36], and that the voltage at node $(n, m)$ given a voltage $V_{0}$ at node $(0,0)$ can be written in terms of Floquet modes $V(m, n)=V_{0} e^{-i \beta(n+m)}$. Enforcing Kirchhoff's laws at every node, and taking $C_{q} \gg C_{g}$, we
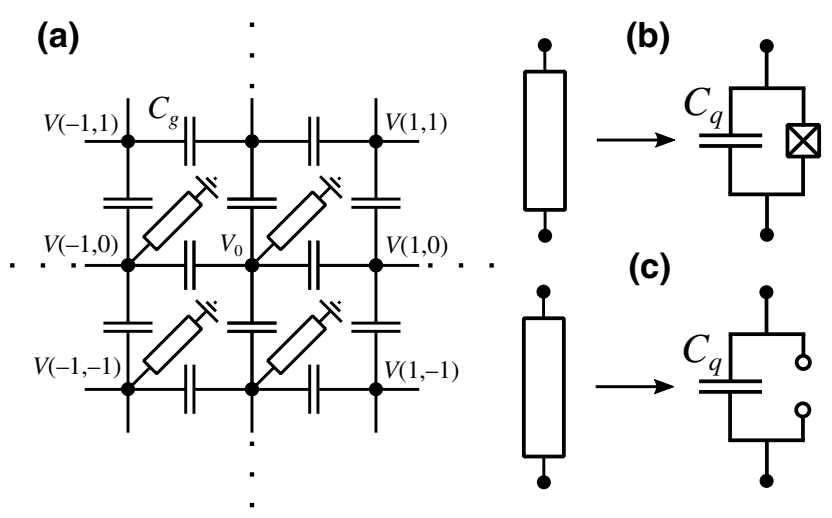

(c)
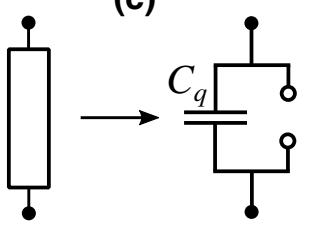

FIG. 12. (a) Circuit model for infinite 2D grid of qubits with nearest-neighbor capacitive couplings. (b) Each shunting element comprises a capacitance $C_{q}$ in parallel with a nonlinear Josephson junction. (c) A linearized version of the circuit, with each Josephson junction replaced by a port.

find

$$
\begin{aligned}
V(n, m) & =V_{0} e^{-(n+m) / \gamma}, \\
\gamma & \approx \frac{1}{\ln \left(\frac{C_{q}}{2 C_{g}}\right)} .
\end{aligned}
$$

Substituting Eqs. (D3) and (D1) into Eq. (C1), and using that $\omega_{q}=1 / \sqrt{L_{i} C_{q}}=1 / \sqrt{L_{j} C_{q}}$, we arrive at

$$
J_{i j}=\frac{1}{2} \omega_{q} e^{-(n+m) / \gamma} .
$$

If qubits are arranged in a square grid with pitch $a$, then the coupling strength depends on the rectilinear distance between qubits, so

$$
\frac{1}{2} \omega_{q} e^{-\sqrt{2} d_{i j} /(a \gamma)} \leq J_{i j} \leq \frac{1}{2} \omega_{q} e^{-d_{i j} /(a \gamma)} .
$$

Thus, in this idealized lumped-element model, circuitmediated cross-talk decays exponentially with spatial separation in a 2D grid of nearest-neighbor capacitively coupled transmon qubits.

[1] Sarah Sheldon, Lev S. Bishop, Easwar Magesan, Stefan Filipp, Jerry M. Chow, and Jay M. Gambetta, Characterizing errors on qubit operations via iterative randomized benchmarking, Phys. Rev. A 93, 012301 (2016).

[2] R. Barends, C. M. Quintana, A. G. Petukhov, Yu Chen, D. Kafri, K. Kechedzhi, R. Collins, O. Naaman, S. Boixo, F. Arute, K. Arya, D. Buell, B. Burkett, Z. Chen, B. Chiaro, and A. Dunsworth, et al., Diabatic Gates for FrequencyTunable Superconducting Qubits, Phys. Rev. Lett. 123, 210501 (2019). 
[3] Johannes Heinsoo, Christian Kraglund Andersen, Ants Remm, Sebastian Krinner, Theodore Walter, Yves Salathé, Simone Gasparinetti, Jean-Claude Besse, Anton Potočnik, Andreas Wallraff, and Christopher Eichler, Rapid HighFidelity Multiplexed Readout of Superconducting Qubits, Phys. Rev. Appl. 10, 034040 (2018).

[4] Matthew D. Reed, Blake R. Johnson, Andrew A. Houck, Leonardo DiCarlo, Jerry M. Chow, David I. Schuster, Luigi Frunzio, and Robert J. Schoelkopf, Fast reset and suppressing spontaneous emission of a superconducting qubit, Appl. Phys. Lett. 96, 203110 (2010).

[5] A. A. Houck, J. A. Schreier, B. R. Johnson, J. M. Chow, Jens Koch, J. M. Gambetta, D. I. Schuster, L. Frunzio, M. H. Devoret, S. M. Girvin, and R. J. Schoelkopf, Controlling the Spontaneous Emission of a Superconducting Transmon Qubit, Phys. Rev. Lett. 101, 080502 (2008).

[6] T. G. McConkey, J. H. Béjanin, C. T. Earnest, C. R. H. McRae, Z. Pagel, J. R. Rinehart, and M. Mariantoni, Mitigating leakage errors due to cavity modes in a superconducting quantum computer, Quantum Sci. Technol. 3, 034004 (2018).

[7] S. Filipp, M. Göppl, J. M. Fink, M. Baur, R. Bianchetti, L. Steffen, and A. Wallraff, Multimode mediated qubitqubit coupling and dark-state symmetries in circuit quantum electrodynamics, Phys. Rev. A 83, 063827 (2011).

[8] Hanhee Paik, D. I. Schuster, Lev S. Bishop, G. Kirchmair, G. Catelani, A. P. Sears, B. R. Johnson, M. J. Reagor, L. Frunzio, L. I. Glazman, S. M. Girvin, M. H. Devoret, and R. J. Schoelkopf, Observation of High Coherence in Josephson Junction Qubits Measured in a ThreeDimensional Circuit qed Architecture, Phys. Rev. Lett. 107, 240501 (2011).

[9] Nicholas T. Bronn, Vivekananda P. Adiga, Salvatore B. Olivadese, and Xian Wu, Jerry M. Chow, and David P. Pappas: High coherence plane breaking packaging for superconducting qubits, Quantum Sci. Technol. 3, 024007 (2018).

[10] James Wenner, Matthew Neeley, Radoslaw C. Bialczak, Michael Lenander, Erik Lucero, Aaron D. O'Connell, Daniel Sank, Haohua Wang, Martin Weides, Andrew N. Cleland, and J. M. Martinis, Wirebond crosstalk and cavity modes in large chip mounts for superconducting qubits, Supercond. Sci. Tech. 24, 065001 (2011).

[11] Jay M. Gambetta, Jerry M. Chow, and Matthias Steffen, Building logical qubits in a superconducting quantum computing system, Npj Quantum Inf. 3, 2 (2017).

[12] Mehrnoosh Vahidpour, William O'Brien, Jon Tyler Whyland, Joel Angeles, Jayss Marshall, Diego Scarabelli, Genya Crossman, Kamal Yadav, Yuvraj Mohan, Catvu Bui, Vijay Rawat, Russ Renzas, Nagesh Vodrahalli, Andrew Bestwick, and Chad Rigetti, Superconducting through-silicon vias for quantum integrated circuits, arXiv:1708.02226 (2017).

[13] Donna-Ruth W. Yost, Mollie E. Schwartz, Justin Mallek, Danna Rosenberg, Corey Stull, Jonilyn L. Yoder, Greg Calusine, Matt Cook, Rabindra Das, Alexandra L. Day, Evan B. Golden, David K. Kim, Alexander Melville, Bethany M. Niedzielski, and Wayne Woods, et al., Solidstate qubits integrated with superconducting throughsilicon vias, Npj Quantum Inf. 6, 1 (2020).
[14] N. A. Nicorovici, R. C. McPhedran, and L. C. Botten, Photonic band gaps for arrays of perfectly conducting cylinders, Phys. Rev. E 52, 1135 (1995).

[15] D. R. Smith, S. Schultz, N. Kroll, M. Sigalas, K. M. Ho, and C. M. Soukoulis, Experimental and theoretical results for a two-dimensional metal photonic band-gap cavity, Appl. Phys. Lett. 65, 645 (1994).

[16] John Preskill, Quantum computing in the NISQ era and beyond, Quantum 2, 79 (2018).

[17] John B. Pendry, A. J. Holden, W. J. Stewart, and I. Youngs, Extremely low Frequency Plasmons in Metallic Mesostructures, Phys. Rev. Lett. 76, 4773 (1996).

[18] P. A. Belov, R. Marques, S. I. Maslovski, I. S. Nefedov, M. Silveirinha, C. R. Simovski, and S. A. Tretyakov, Strong spatial dispersion in wire media in the very large wavelength limit, Phys. Rev. B 67, 113103 (2003).

[19] P. A. Belov, S. A. Tretyakov, and A. J. Viitanen, Dispersion and reflection properties of artificial media formed by regular lattices of ideally conducting wires, J. Electromagnet. Wave. 16, 1153 (2002).

[20] A. Krynkin and P. McIver, Approximations to wave propagation through a lattice of dirichlet scatterers, Waves Random Complex Media 19, 347 (2009).

[21] Conal E. Murray and D. W. Abraham, Predicting substrate resonance mode frequency shifts using conductive, through-substrate vias, Appl. Phys. Lett. 108, 084101 (2016).

[22] John B. Pendry, A. J. Holden, D. J. Robbins, and W. J. Stewart, Low frequency plasmons in thin-wire structures, J. Phys. Condens. Matter 10, 4785 (1998).

[23] Richard Remski, Analysis of photonic bandgap surfaces using Ansoft HFSS, Microw. J. 43, 190 (2000).

[24] Michael J. Hartmann, Fernando G. S. L. Brandao, and Martin B. Plenio: Strongly interacting polaritons in coupled arrays of cavities, Nat. Phys. 2, 849 (2006).

[25] D. E. Nagle, E. A. Knapp, and B. C. Knapp, Coupled resonator model for standing wave accelerator tanks, Rev. Sci. Instrum. 38, 1583 (1967).

[26] Thomas P. Wangler, RF Linear Accelerators (John Wiley \& Sons, Hoboken, 2008).

[27] Nathan Marcuvitz, Waveguide Handbook (IET, New York, 1951), p. 21.

[28] Tao Shi, Ying-Hai Wu, Alejandro González-Tudela, and J. Ignacio Cirac, Bound States in Boson Impurity Models, Phys. Rev. X 6, 021027 (2016).

[29] James S. Douglas, Hessam Habibian, C.-L. Hung, Alexey V. Gorshkov, H. Jeff Kimble, and Darrick E. Chang, Quantum many-body models with cold atoms coupled to photonic crystals, Nat. Photonics 9, 326 (2015).

[30] Alejandro González-Tudela, C.-L. Hung, Darrick E. Chang, J. Ignacio Cirac, and H. J. Kimble, Subwavelength vacuum lattices and atom-atom interactions in twodimensional photonic crystals, Nat. Photonics 9, 320 (2015).

[31] J. Rahamim, T. Behrle, M. J. Peterer, A. Patterson, P. A. Spring, T. Tsunoda, R. Manenti, G. Tancredi, and P. J. Leek, Double-sided coaxial circuit QED with out-of-plane wiring, Appl. Phys. Lett. 110, 222602 (2017). 
[32] A. D. Patterson, J. Rahamim, T. Tsunoda, P. A. Spring, S. Jebari, K. Ratter, M. Mergenthaler, G. Tancredi, B. Vlastakis, M. Esposito, and P. J. Leek, Calibration of a Cross-Resonance Two-Qubit Gate between Directly Coupled Transmons, Phys. Rev. Appl. 12, 064013 (2019).

[33] Firat Solgun, David P. DiVincenzo, and Jay M. Gambetta, Simple impedance response formulas for the dispersive interaction rates in the effective hamiltonians of low anharmonicity superconducting qubits, IEEE Trans. Microw. Theory Tech. 67, 928 (2019).
[34] William Hart Hayt, Jack Ellsworth Kemmerly, and Steven M. Durbin, Engineering Circuit Analysis (McGraw-Hill, New York, 1978), Vol. 214.

[35] L. Losonczi, Eigenvalues and eigenvectors of some tridiagonal matrices, Acta. Math. Hung. 60, 309 (1992).

[36] Rubaiyat Islam, Michael Zedler, and George V. Eleftheriades, Modal analysis and wave propagation in finite 2D transmission-line metamaterials, IEEE Trans. Antennas Propag. Trans. Antennas Propag. 59, 1562 (2011). 